\title{
DIRECTION FINDING ALGORITHMS FOR TIME REVERSAL MIMO RADARS
}

\author{
Foroohar Foroozan ${ }^{*}$, Amir Asif* ${ }^{*}$ Yunwai Jin ${ }^{\dagger}$, and José M.F. Moura \\ ${ }^{*}$ Computer Science and Engineering, York University, Toronto, ON, Canada \\ ${ }^{\dagger}$ Engineering and Aviation Sciences, University of Maryland Eastern Shore, Princess Anne, MD, USA \\ ${ }^{\ddagger}$ Electrical and Computer Engineering, Carnegie Mellon University, Pittsburgh, PA, USA
}

\begin{abstract}
A time reversal (TR) based direction of arrival (DOA) estimation framework for multiple-input/multiple-output (MIMO) radars is presented. We develop minimum variance distortionless response (MVDR) and multiple signal classification (MUSIC) based DOA estimators for the TR/MIMO setup. The TR/MIMO estimation algorithms outperform their conventional counterparts in: $(i)$ analytical Cramér Rao Bounds (CRB) comparisons, and; ( $i i)$ numerical Monte Carlo simulations for a range of signal to noise ratios that we tested.
\end{abstract}

Index Terms - Time Reversal, Cramér-Rao Bounds, DOA Estimation, Localization, MIMO, and Multipath.

\section{INTRODUCTION}

The paper explores the potential of using an adaptive waveform design technique known as time reversal (TR) in conjunction with multiple-input/multiple-output (MIMO) radars [1] to design new source localization algorithms. Unlike the standard phased-array radars, which transmit scaled versions of a single waveform, a MIMO radar system has the capacity to transmit multiple and possibly different probing signals simultaneously. This additional degree of freedom, supplemented by the ability to design the probing waveforms according to the channel characteristics, is used to optimize a desired criteria, such as enhancing detection performance, increasing clutter/interference rejection, or improving metric accuracy.

To date, most of the work on the probing signal design in MIMO radars has focused on non-adaptive waveform shaping [2], where a particular waveform design is strictly followed throughout the estimation process. By incorporating TR in MIMO radars, the paper employs an adaptive waveform reshaping mechanism, which conforms the probing waveforms to the propagation environment. The difference in MIMO and TR/MIMO radars is, therefore, obvious. First, while the conventional MIMO radar uses a pretrained set of probing signals (generally orthogonal to each other), TR/MIMO radar adapts the transmission waveforms to the multipath environment and thus provides a straightforward procedure for adaptive waveform coding based on the channel characteristics. Recently, Moura et al. [3, 4] have introduced TR/MIMO radars for target detection. This paper extends the TR/MIMO radar framework, we believe for the first time, to target localization. A second distinction of TR/MIMO radar lies in its positive treatment of multipath - normally considered detrimental in conventional radar systems.

The novel contributions of the paper, therefore, are: (i) Applying TR/MIMO to estimate the direction of arrival (DOA) of a target

The work was supported in part by Natural Science and Engineering Research Council (NSERC), Canada under Grant No. 228415-2010. Y. Jin was supported in part by a 2010 Air Force Summer Faculty Fellowship award. embedded in a highly cluttered environment, and; (ii) Deriving the Cramér-Rao bounds (CRB) for the proposed TR/MIMO DOA estimator. Combined with the traditional DOA approaches based on minimum variance distortionless response (MVDR) and multiple signal classification (MUSIC), the TR/MIMO estimator significantly improves the estimation performance of these algorithms across a range of signal to noise ratios (SNR) tested in our Monte Carlo simulations. In terms of organization, Section 2 formulates the problem for both conventional MIMO and TR/MIMO frameworks. Section 3 reviews the classical DOA estimators (MUSIC and MVDR) used as a proof of concept for the MIMO and TR/MIMO radars, and also derives their CRBs. Section 4 compares their performances through Monte Carlo simulations. Finally, Section 5 concludes the paper.

\section{SYSTEM MODEL}

Consider a monostatic radar consisting of two colocated arrays with $P_{T}$ transmit and $P_{R}$ receive elements. Element $i$ of the transmit array probes the channel with a bandpass signal $f_{i}(t) e^{j \omega_{c} t}$, where $f_{i}(t)$ is the complex baseband envelope of the probing signal and $\omega_{c}$ is the carrier angular frequency. The backscatter from a point target observed at the $j$ 'th receiver, $\left(1 \leq j \leq P_{R}\right)$, is given by

$$
r_{j}(t)=\sum_{i=1}^{P_{T}} X_{i j} f_{i}\left(t-\tilde{\tau}_{i}^{T}-\tilde{\tau}_{j}^{R}\right) e^{j \omega_{c}\left(t-\tilde{\tau}_{i}^{T}-\tilde{\tau}_{j}^{R}\right)}+r_{c}(t)+v(t)
$$

where $\tilde{\tau}_{i}^{T}=\left(r_{0} / c\right)+\tau_{i}^{T}(\theta)$ is the propagation delay between element $i$ of the transmit array and the far-field target at range $r_{0}$ along direction $\theta$ and $c$ denotes the propagation speed in the medium. Similarly, $\tilde{\tau}_{j}^{R}=\left(r_{0} / c\right)+\tau_{j}^{R}(\theta)$ is the propagation delay between element $j$ of the receive array and the target. Terms $\left\{\tau_{i}^{T}(\theta), \tau_{j}^{R}(\theta)\right\}$ are, respectively, the inter-element delays between the $i$ 'th transmit and $j$ 'th receive elements from $r_{0}$. The complex term $X_{i j}$ corresponds to the path loss including the target's reflection coefficient. The clutter response and noise are denoted, respectively, by $r_{c}(t)$ and $v(t)$. The signal $r_{j}(t)$ is down-converted to baseband then the Fourier transform is performed, which gives

$$
\begin{aligned}
R_{j}\left(e^{j \omega}\right)=\sum_{i=1}^{P_{T}} & {\left[X_{i j} e^{-j\left(\omega+\omega_{c}\right)\left(2 r_{0} / c+\tau_{i}^{T}(\theta)+\tau_{j}^{R}(\theta)\right)} F_{i}\left(e^{j \omega}\right)\right] } \\
& +R_{c}^{\prime}\left(e^{j \omega}\right)+V^{\prime}\left(e^{j \omega}\right),
\end{aligned}
$$

where $F_{i}\left(e^{j \omega}\right)$ is the discrete time Fourier transform (DTFT) of $f_{i}(t)$. Notations $R_{c}^{\prime}\left(e^{j \omega}\right)$, and $V^{\prime}\left(e^{j \omega}\right)$ are, respectively, the frequency downconverted versions of the DTFTs of $r_{c}(t)$ and $v(t)$.

Conventional MIMO Radar: is shown in Fig. 1 and is based on the following steps. 
1. Conventional Probing: In Eq. (2), we define the attenuation coefficient $X=X_{i j} \exp \left(-j 2 \omega_{c} r_{0} / c\right)$. Based on the far-field assumption, the path losses $X_{i j}$ are assumed identical for all pairs of transmit and receive elements for the backscatter travelling via the direct path. In the vector-matrix form, Eq. (2) is, therefore, given by

$$
\mathbf{r}\left(e^{j \omega}\right)=X e^{-j 2 \omega r_{0} / c} \mathbf{A}(\theta) \mathbf{f}\left(e^{j \omega}\right)+\mathbf{r}_{c}\left(e^{j \omega}\right)+\mathbf{v}\left(e^{j \omega}\right)(3)
$$

where $\mathbf{r}\left(e^{j \omega}\right)$ is the ordered vector for $R_{j}\left(e^{j \omega}\right) ; \mathbf{r}_{c}\left(e^{j \omega}\right)$ and $\mathbf{v}\left(e^{j \omega}\right)$ are, respectively, the clutter and noise vectors; $\mathbf{A}(\theta)$ $=\boldsymbol{a}_{R}(\theta) \boldsymbol{a}_{T}^{T}(\theta)$ is the transmit-receive steering matrix with

$$
\begin{aligned}
\boldsymbol{a}_{T}(\theta) & =\left[e^{-j\left(\omega+\omega_{c}\right) \tau_{1}^{T}(\theta)}, \cdots, e^{-j\left(\omega+\omega_{c}\right) \tau_{P_{T}}^{T}(\theta)}\right]^{T}, \\
\boldsymbol{a}_{R}(\theta) & =\left[e^{-j\left(\omega+\omega_{c}\right) \tau_{1}^{R}(\theta)}, \cdots, e^{-j\left(\omega+\omega_{c}\right) \tau_{P_{R}}^{R}(\theta)}\right]^{T}, \\
\text { and } \mathbf{f}\left(e^{j \omega}\right) & =\left[F_{1}\left(e^{j \omega}\right), \cdots, F_{P_{T}}\left(e^{j \omega}\right)\right]^{T} .
\end{aligned}
$$

With interference backscatters part of the clutter, Eq. (3) is

$$
\mathbf{r}\left(e^{j \omega}\right)=\underbrace{\mathbf{H}\left(e^{j \omega}\right) \mathbf{f}\left(e^{j \omega}\right)}_{\mathbf{r}_{t}\left(e^{j \omega}\right)}+\underbrace{\mathbf{H}_{c}\left(e^{j \omega}\right) \mathbf{f}\left(e^{j \omega}\right)+\mathbf{v}\left(e^{j \omega}\right)}_{\mathbf{r}_{z}\left(e^{j \omega}\right)},
$$

where $\mathbf{H}\left(e^{j \omega}\right)=X e^{-j 2 \omega r_{0} / c} \mathbf{A}(\theta)$ contains the unknown parameters and $\mathbf{H}_{c}\left(e^{j \omega}\right)$ is the clutter frequency response. In $\mathbf{r}_{z}\left(e^{j \omega}\right)$, the clutter component is $\mathbf{r}_{c}\left(e^{j \omega}\right)=\mathbf{H}_{c}\left(e^{j \omega}\right) \mathbf{f}\left(e^{j \omega}\right)$.

2. Conventional Clutter Probing: A training stage in the absence of the target estimates the statistics of the clutter and also models the clutter frequency response $\mathbf{H}_{c}\left(e^{j \omega}\right)$. The channel is probed with $\mathbf{f}\left(e^{j \omega}\right)$ and the clutter backscatters $\mathbf{r}_{c}\left(e^{j \omega}\right)$ are recorded. In modeling $\mathbf{H}_{c}\left(e^{j \omega}\right)$, we follow the approach presented in [4,5], where the clutter is characterized in the spatial and spectral domains, and represented as a multivariate complex Gaussian random process. The covariance matrix of the clutter returns $\mathbf{R}_{\mathbf{r}_{c}}$ is estimated in this step.

3. Conventional Clutter Suppression: With the noise component $\mathbf{v} \sim \mathcal{C} \mathcal{N}\left(0, \sigma_{n}^{2} \mathbf{I}_{P_{R}}\right)$, the covariance of the clutter-noise component $\mathbf{r}_{z}$ is $\mathbf{R}_{\mathbf{r}_{z}}=\mathbf{R}_{\mathbf{r}_{c}}+\sigma_{n}^{2} \mathbf{I}_{P_{R}}$. Noting that the clutter-noise covariance matrix $\mathbf{R}_{\mathbf{r}_{z}}$ is a positive definite matrix, a whitening filter is used to convert the clutter-noise component to white noise. The whitened observation is given by

$$
\tilde{\mathbf{r}}\left(e^{j \omega}\right)=\underbrace{\mathbf{R}_{\mathbf{r}_{z}}^{-\frac{1}{2}} \mathbf{H}\left(e^{j \omega}\right) \mathbf{f}\left(e^{j \omega}\right)}_{\tilde{\mathbf{r}}_{t}\left(e^{j \omega}\right)}+\underbrace{\mathbf{R}_{\mathbf{r}_{z}}^{-\frac{1}{2}} \mathbf{r}_{z}\left(e^{j \omega}\right)}_{\tilde{\mathbf{r}}_{z}\left(e^{j \omega}\right)},
$$

with the whitened clutter-noise term $\tilde{\mathbf{r}}_{z}\left(e^{j \omega}\right) \sim \mathcal{C N}\left(0, \sigma_{z}^{2} \mathbf{I}_{P_{R}}\right)$. The whitened channel response $\tilde{\mathbf{H}}\left(e^{j \omega}\right)=\mathbf{R}_{\mathbf{r}_{z}}^{-1 / 2} \mathbf{H}\left(e^{j \omega}\right)$.

4. Conventional MIMO Matched Filtering: The wideband observations are decomposed into $Q=B / B_{c}$ narrowband bins using either the DTFT or filter banks before applying a narrowband localization algorithm, where $B_{c}$ is the coherence bandwidth of the multipath channel and $B$ the system bandwidth. The output $\mathbf{B}_{\tilde{\mathbf{r}}}$ of the filter matched to the probing signal $\mathbf{f}\left(e^{j \omega_{q}}\right)$ when applied to the MIMO observation $\tilde{\mathbf{r}}\left(e^{j \omega_{q}}\right)$ provides sufficient statistics for estimating the unknown parameters [2]. The output of the matched filter is

$$
\begin{aligned}
\mathbf{B}_{\tilde{\mathbf{r}}} & =\sum_{q=0}^{Q-1}\left[X e^{-j 2 \omega_{q} r_{0} / c} \mathbf{R}_{\mathbf{r}_{z}}^{-\frac{1}{2}} \mathbf{A}(\theta) \mathbf{f}\left(e^{j \omega_{q}}\right) \mathbf{f}^{H}\left(e^{j \omega_{q}}\right)\right. \\
& \left.+\tilde{\mathbf{r}}_{z}\left(e^{j \omega_{q}}\right) \mathbf{f}^{H}\left(e^{j \omega_{q}}\right)\right]
\end{aligned}
$$

The conventional radar uses (6) to estimate the DOA $\theta$.

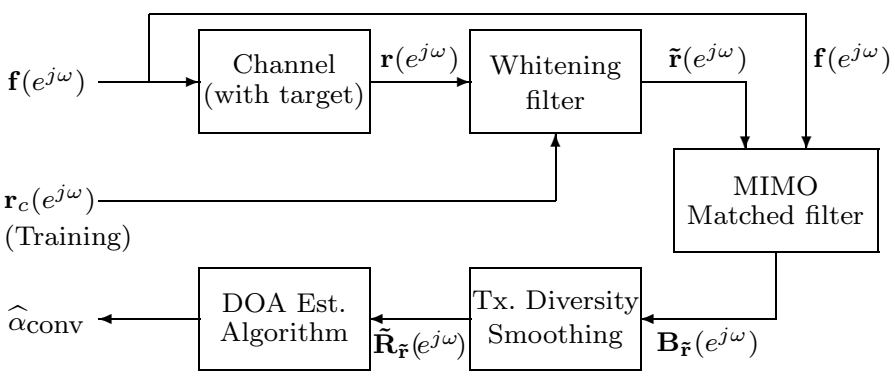

Fig. 1. Schematic diagram for the conventional MIMO DOA radar.

TR/MIMO Radar: In addition to Steps 1-3, the TR/MIMO DOA estimation includes the following steps. Step 4 is not needed here.

5. Time Reversal Probing: The whitened backscatters $\tilde{\mathbf{r}}\left(e^{j \omega}\right)$ from Eq. (5) in Step 3 are time-reversed, energy normalized, and are used to probe the channel a second time. The TR probing signal is given by $\mathbf{f}_{T R}\left(e^{j \omega}\right)=g\left[\tilde{\mathbf{r}}\left(e^{j \omega}\right)\right]^{*}$, where $g=\sqrt{\int\left\|\mathbf{f}\left(e^{j \omega}\right)\right\|^{2} d \omega / \int\left\|\tilde{\mathbf{r}}\left(e^{j \omega}\right)\right\|^{2} d \omega}$ is the TR normalization constant with respect to the whitened backscatter $\tilde{\mathbf{r}}\left(e^{j \omega}\right)$. The TR backscatter observations are

$$
\mathbf{p}\left(e^{j \omega}\right)=g \mathbf{H}^{T}\left(e^{j \omega}, \boldsymbol{\alpha}\right) \tilde{\mathbf{r}}^{*}\left(e^{j \omega}\right)+\mathbf{p}_{c}\left(e^{j \omega}\right)+\mathbf{p}_{n}\left(e^{j \omega}\right) .
$$

Substituting Eq. (5) in Eq. (7) gives

$$
\begin{aligned}
\mathbf{p}\left(e^{j \omega}\right) & =\underbrace{g \mathbf{H}^{T}\left(e^{j \omega}\right) \tilde{\mathbf{H}}^{*}\left(e^{j \omega}\right) \mathbf{f}^{*}\left(e^{j \omega}\right)}_{\mathbf{p}_{t}\left(e^{j \omega}\right)} \\
+ & \underbrace{g \mathbf{H}^{T}\left(e^{j \omega}\right) \tilde{\mathbf{r}}_{z}^{*}\left(e^{j \omega}\right)+\mathbf{H}_{c}^{T}\left(e^{j \omega}\right) \tilde{\mathbf{r}}^{*}\left(e^{j \omega}\right)+\mathbf{p}_{n}\left(e^{j \omega}\right)}_{\mathbf{p}_{z}\left(e^{j \omega}\right)} .
\end{aligned}
$$

In $\mathbf{p}_{z}\left(e^{j \omega}\right)$, the clutter component $\mathbf{p}_{c}\left(e^{j \omega}\right)=\mathbf{H}_{c}^{T}\left(e^{j \omega}\right) \tilde{\mathbf{r}}^{*}\left(e^{j \omega}\right)$.

6. TR Training: The TR training step time reverses the clutter returns $\mathbf{r}_{c}\left(e^{j \omega}\right)$ obtained from the conventional clutter probing stage and probes the channel in the presence of the target with $\mathbf{r}_{c}^{*}\left(e^{j \omega}\right)$. The backscatter of this probing is given by

$$
\mathbf{p}_{c t}\left(e^{j \omega}\right)=g_{c}\left[\mathbf{H}\left(e^{j \omega}\right)+\mathbf{H}_{c}\left(e^{j \omega}\right)\right]^{T} \mathbf{r}_{c}^{*}\left(e^{j \omega}\right)+\mathbf{n}\left(e^{j \omega}\right),
$$

where $g_{c}=\sqrt{\int\left\|\mathbf{f}\left(e^{j \omega}\right)\right\|^{2} d \omega / \int\left\|\mathbf{r}_{c}\left(e^{j \omega}\right)\right\|^{2} d \omega}$ is the normalization constant with respect to the clutter returns. Since an estimate of $\mathbf{H}_{c}\left(e^{j \omega}\right)$ is available (Step 2), term $g_{c} \mathbf{H}_{c}^{T} \mathbf{r}_{c}^{*}$ is subtracted out from $\mathbf{p}_{c t}$ in Eq. (9).

7. TR Clutter Suppression: The whitening filter for the TR stage is based on Eq. (8), which has two clutter components: $\mathbf{H}^{T}\left(e^{j \omega}\right) \tilde{\mathbf{r}}_{z}^{*}\left(e^{j \omega}\right)$ and $\mathbf{H}_{c}^{T}\left(e^{j \omega}\right) \tilde{\mathbf{r}}^{*}\left(e^{j \omega}\right)$. An estimate of the clutter component in the first term is obtained from Step 6, while a determination of the clutter for the second term is obtained by estimating the clutter frequency response $\mathbf{H}_{c}$ from the conventional clutter probing stage. We follow the same whitening approach as described earlier for the conventional MIMO. The whitened TR observations are then given by

$$
\tilde{\mathbf{p}}\left(e^{j \omega}\right)=\underbrace{\mathbf{R}_{\mathbf{p}_{z}}^{-\frac{1}{2}} \mathbf{p}_{t}\left(e^{j \omega}\right)}_{\tilde{\mathbf{p}}_{t}\left(e^{j \omega}\right)}+\underbrace{\mathbf{R}_{\mathbf{p}_{z}}^{-\frac{1}{2}} \mathbf{p}_{z}\left(e^{j \omega}\right)}_{\tilde{\mathbf{p}}_{z}\left(e^{j \omega}\right)}
$$


with the whitened clutter-noise term $\tilde{\mathbf{p}}_{z}\left(e^{j \omega}\right) \sim \mathcal{C N}\left(0, \sigma_{w}^{2} \mathbf{I}_{P_{T}}\right)$. Using Eq. (8), the whitened return $\tilde{\mathbf{p}}\left(e^{j \omega}\right)$ in the TR step is

$\tilde{\mathbf{p}}\left(e^{j \omega}\right)=g \mathbf{R}_{\mathbf{p}_{z}}^{-\frac{1}{2}} \mathbf{H}^{T}\left(e^{j \omega}\right) \tilde{\mathbf{H}}^{*}\left(e^{j \omega}\right) \mathbf{f}^{*}\left(e^{j \omega}\right)+\tilde{\mathbf{p}}_{z}\left(e^{j \omega}\right)$,

with the whitened TR matrix $\tilde{\mathbf{T}}\left(e^{j \omega}\right)=\mathbf{R}_{\mathbf{p}_{z}}^{-\frac{1}{2}} \mathbf{H}^{T}\left(e^{j \omega}\right) \tilde{\mathbf{H}}^{*}\left(e^{j \omega}\right)$.

8. TR/MIMO Matched Filtering: Next, we apply the matched filter to the TR observations $\tilde{\mathbf{p}}\left(e^{j \omega}\right)$ in order to formulate the sufficient statistics matrix $\mathbf{B}_{\tilde{\mathbf{p}}}$ for the TR/MIMO radar. The TR probing waveforms $\tilde{\mathbf{r}}^{*}\left(e^{j \omega}\right)$ can not be used directly to design the matched filter as these are not necessarily orthogonal and will result in the TR sufficient statistics $\mathbf{B}_{\tilde{\mathbf{p}}}$ that are statistically dependent. So, we transform $\tilde{\mathbf{r}}^{*}\left(e^{j \omega}\right)$ to a vector of signals [6] as follows

$$
\tilde{\mathbf{f}}_{T R}=\mathbf{V}^{-1 / 2} \mathbf{U}^{H} \tilde{\mathbf{r}}^{*}\left(e^{j \omega}\right),
$$

in which $\mathbf{U}$ and $\mathbf{V}$ are, respectively, the matrices of eigenvectors and eigenvalues of the correlation matrix of $\tilde{\mathbf{r}}^{*}$. The resulting waveforms $\tilde{\mathbf{f}}_{T R}$ are used to design the matched filter, which results in the TR/MIMO statistics

$$
\begin{aligned}
& \mathbf{B}_{\tilde{\mathbf{p}}}=\int \tilde{\mathbf{p}}\left(e^{j \omega}\right) \tilde{\mathbf{f}}_{T R}^{H}\left(e^{j \omega}\right) d \omega \\
& \quad=\int \tilde{\mathbf{p}}_{z}\left(e^{j \omega}\right) \tilde{\mathbf{f}}_{T R}^{H}\left(e^{j \omega}\right) d \omega+g \int \tilde{\mathbf{T}}\left(e^{j \omega}\right) \mathbf{f}^{*}\left(e^{j \omega}\right) \tilde{\mathbf{f}}_{T R}^{H}\left(e^{j \omega}\right) d \omega
\end{aligned}
$$

The conventional MIMO radar is based on Eq. (6), while the TR/ MIMO radar, shown in Fig. 2, uses Eq. (13) for target localization.

\section{MIMO DIRECTION ESTIMATION}

As a proof of concept, two classical DOA estimators, MUSIC and MVDR, are applied to the conventional and TR observations modeled, respectively, in Eqs. (6) and (13). Recall that the TR probing signals are reshaped and adaptively adjusted to the scattering properties of the medium. In order to de-correlate the time reversed backscatters, the TR/MIMO radar requires a preprocessing step based on the Transmission Diversity Smoothing (TDS) algorithm [6], which is applied to the covariance matrix of the TR model.

$$
\tilde{\mathbf{R}}_{\tilde{\mathbf{p}}}=\left(1 / P_{R}\right) \sum_{i=1}^{P_{R}}\left\{\mathbf{R}_{\tilde{\mathbf{p}}}\right\}_{i},
$$

where $\left\{\mathbf{R}_{\tilde{\mathbf{p}}}\right\}_{i}$ is the covariance matrix for the $i$ 'th column of the TR sufficient statistics $\mathbf{B}_{\tilde{\mathbf{p}}}$ (Eq. (13)). This is equivalent to smoothing the correlation matrices obtained from each column of the matrix $\mathbf{B}_{\tilde{\mathbf{p}}}$.

TR/MIMO MVDR: Combining results from different bins, the geometrically averaged wideband TR MVDR has the power spectrum

$$
\mathbb{Q}_{M V}^{T R}(\theta)=(1 / Q) \prod_{q=1}^{Q}\left\{\boldsymbol{a}_{T}^{H}(\theta) \tilde{\mathbf{R}}_{\tilde{\mathbf{p}}}^{-1}\left(e^{j \omega_{q}}\right) \boldsymbol{a}_{T}(\theta)\right\}^{-1}
$$

TR/MIMO MUSIC: Assuming that the covariance matrix $\tilde{\mathbf{R}}_{\tilde{\mathbf{p}}}$ has the eigen decomposition

$$
\tilde{\mathbf{R}}_{\tilde{\mathbf{p}}}=\mathbf{U}_{\tilde{\mathbf{p}}}^{s} \mathbf{V}_{\tilde{\mathbf{p}}}^{s}\left(\mathbf{U}_{\tilde{\mathbf{p}}}^{s}\right)^{H}+\sigma_{w}^{2} \mathbf{U}_{\tilde{\mathbf{p}}}^{n}\left(\mathbf{U}_{\tilde{\mathbf{p}}}^{n}\right)^{H},
$$

where $\mathbf{U}_{\tilde{\mathbf{p}}}^{s}$ and $\mathbf{V}_{\tilde{\mathbf{p}}}^{s}$ are, respectively, the eigenvector and eigenvalue matrix of $\tilde{\mathbf{R}}_{\tilde{\mathbf{p}}}$ in the signal subspace and $\mathbf{U}_{\tilde{\mathbf{p}}}^{n}$ is the eigenvector of $\tilde{\mathbf{R}}_{\tilde{\mathbf{p}}}$ in the noise subspace, the wideband TR/MIMO MUSIC spectrum is

$$
\mathbb{Q}_{M U}^{T R}(\theta)=(1 / Q) \prod_{q=1}^{Q}\left\{\frac{\boldsymbol{a}_{T}^{H}(\theta) \mathbf{U}_{\tilde{\mathbf{p}}}^{n}\left(\mathbf{U}_{\tilde{\mathbf{p}}}^{n}\right)^{H} \boldsymbol{a}_{T}(\theta)}{\boldsymbol{a}_{T}^{H}(\theta) \boldsymbol{a}_{T}(\theta)}\right\}^{-1}
$$

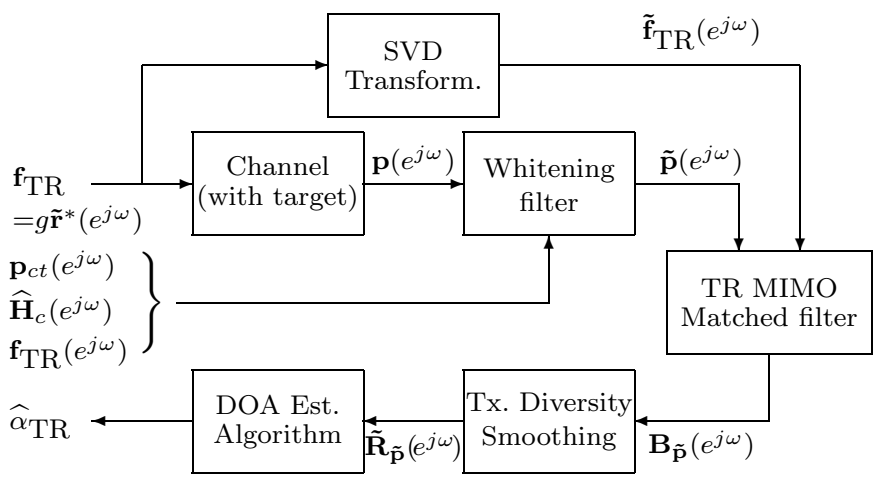

Fig. 2. Schematic diagram for the TR/MIMO DOA radar.

Conventional MIMO Direction Estimation: In order to compare the TR/MIMO radar with its MIMO counterpart, the MVDR and MUSIC DOA algorithms are implemented for the MIMO radar. We follow the procedure for TR/MIMO expressed in Eqs. (15) and (17), but instead of the TR covariance matrix $\tilde{\mathbf{R}}_{\tilde{\mathbf{p}}}$, the covariance matrix $\mathbf{R}_{\tilde{\mathbf{r}}}$ based on the conventional observations is processed by the TDS step (similar to Eq. (14)) and the resulting matrix is used.

3.1. Performance Bounds: Extending the CRBs [7] for single-input multiple-output (SIMO) radars to MIMO radars, the following CRB expressions for unknown parameter $\boldsymbol{\alpha}=\left[r_{0}, \theta\right]^{T}$ are derived.

Conventional MIMO DOA Estimator:

$$
\mathrm{CRB}_{\mathrm{CV}}(\boldsymbol{\alpha})^{-1}=\frac{N}{\pi \sigma_{z}^{2}} \times \Re\left\{\int \tilde{\mathbf{f}}^{H}\left(e^{j \omega}\right) \mathbf{D}^{H} \mathbf{D} \tilde{\mathbf{f}}\left(e^{j \omega}\right) d \omega\right\}(18)
$$

TR MIMO DOA Estimator:

$$
\mathrm{CRB}_{\mathrm{TR}}(\boldsymbol{\alpha})^{-1}=\frac{g^{2} N}{\pi \sigma_{w}^{2}} \times \Re\left\{\int \tilde{\mathbf{f}}^{T}\left(e^{j \omega}\right) \mathbf{E}^{H} \mathbf{E} \tilde{\mathbf{f}}^{*}\left(e^{j \omega}\right) d \omega\right\}(19)
$$

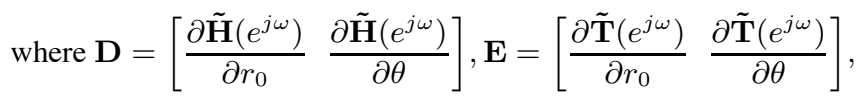

and $\tilde{\mathbf{f}}=\mathbf{f} \otimes \mathbf{I}_{2}$. In the above expressions, $N$ is the total number of snapshots. The proofs are based on the methodology introduced in [8] but omitted here due to lack of space.

\section{NUMERICAL SIMULATIONS}

In this section, we carry out Monte Carlo simulations to evaluate the performance of the proposed TR/MIMO DOA estimators. A MIMO radar system based on two colocated arrays with $P_{T}=P_{R}=10$ antennae elements with half-wavelength inter-element spacing is used. For the probing waveforms, we follow [4] and use the phase coding scheme $F_{i}\left(e^{j \omega_{q}}\right)=e^{j 2 \pi i q / Q} F\left(e^{j \omega_{q}}\right)$, for $(1 \leq i \leq 10)$ and $(0 \leq q \leq Q-1)$ with $Q=10$ bins, where $F\left(e^{j \omega_{q}}\right)$ is the DTFT of the linear frequency modulated signal

$$
f(t)=\frac{1}{\sqrt{\tau}_{0}} \operatorname{Rect}\left(\frac{t}{\tau_{0}}\right) e^{j \pi \mu t^{2}} e^{j \omega_{c} t} .
$$

The angular frequency $\omega_{c}=2 \pi f_{c}$ with the base chirp frequency $f_{c}=5 \mathrm{GHz}$. The width of the pulse $\tau_{0}$ is set to $50 \mu \mathrm{s}$ and parameter $\mu=B / \tau_{0}$ with the bandwidth $B$ set to $100 \mathrm{MHz}$. The setup includes a single target located at $\theta=40^{\circ}$ with a range of $1 \mathrm{~km}$ embedded in a highly cluttered environment. The clutter model follows the complex Gaussian approach characterized in both spatial 


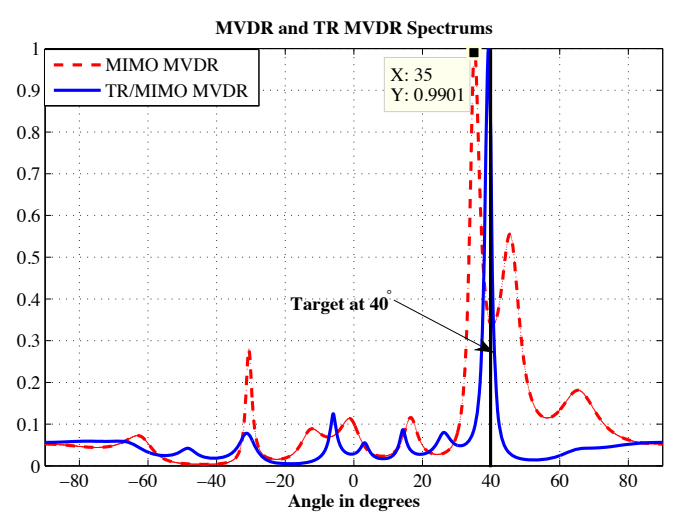

(a)

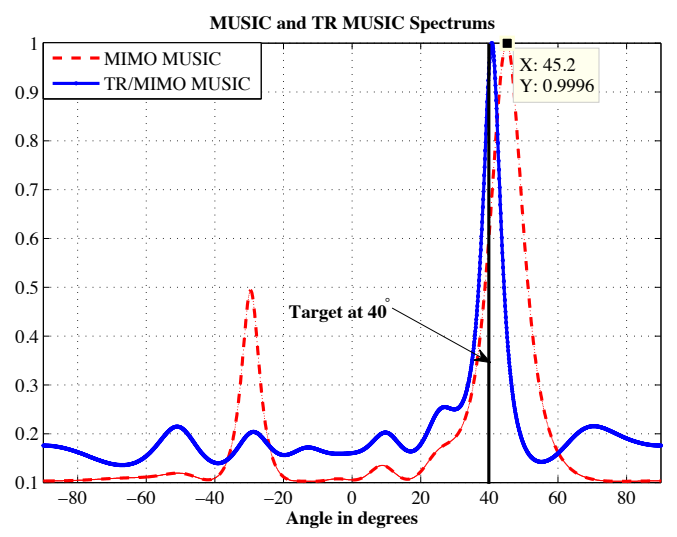

(b)

Fig. 3. (a) MIMO MVDR and (b) MIMO MUSIC DOA estimates for a target located at $40^{\circ}$ and two interference sources at $\left\{50^{\circ},-30^{\circ}\right\}$. The SNR is set to $10 \mathrm{~dB}$ with additional Gaussian clutter having the signal to clutter ratio (SCR) of $10 \mathrm{~dB}$. The outputs of the TR/DOA estimators are shown with firm lines, while those of the conventional estimators are shown with dotted lines.

and spectral domains suggested in [5]. In addition to the clutter and noise model included in Eq. (4) for the conventional MIMO radar, two interference sources located at $\left\{50^{\circ},-30^{\circ}\right\}$ transmit random binary phase shift keying (BPSK) symbols with high power. The received signals have $N=2500$ snapshots, each corrupted with a zero-mean white Gaussian noise, whose variance is adjusted to provide the desired range of SNRs. The TR received waveforms follow the approach presented in Section 2 with the addition of jammers similar to the ones introduced in the conventional MIMO radar. The MVDR and MUSIC algorithms are used to plot the pseudospectrum in Fig. 3. While both the MIMO/MVDR and MIMO/MUSIC are unable to locate the true DOA of the target at $40^{\circ}$ and deviate from this value because of the presence of clutter and interference source at $50^{\circ}$, their TR counterparts locate the target with a high accuracy. Further, the MUSIC and MVDR algorithms based on the conventional MIMO suffer from higher sidelobes and wider mainlobes (lower resolutions). On the other hand, the DOA estimators based on TR/MIMO offer higher immunity to interference. Fig. 4 plots the root-mean-square error (RMSE) obtained from a Monte Carlo simulation of 1000 runs for different SNRs with the SCR set to $10 \mathrm{~dB}$. The TR/MIMO DOA estimators exhibits considerably lower RMSE at all SNRs. The figure also shows the CRBs (obtained by discretiz-

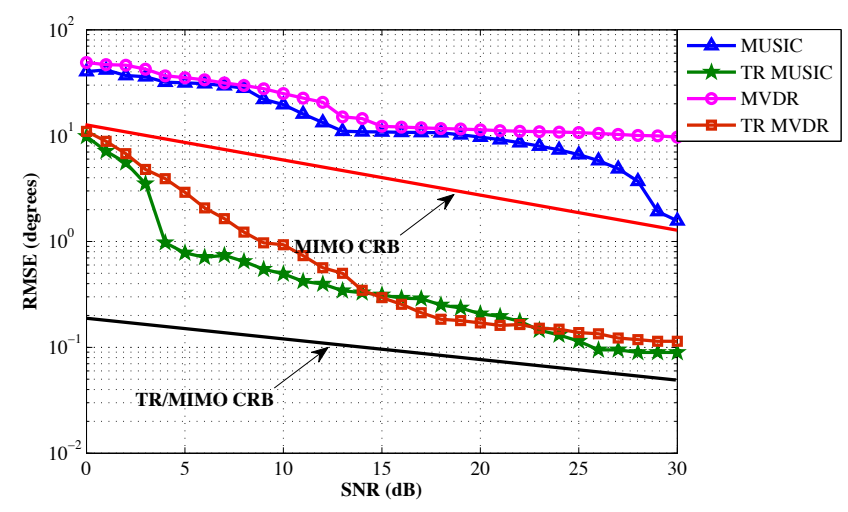

Fig. 4. Performance curves for the MIMO and TR/MIMO DOA estimators. For comparison, the plots for the CRBs are also included.

ing Eqs. (18) and (19) and running numerical simulations to compute terms $\mathbf{D}^{H} \mathbf{D}$ and $\mathbf{E}^{H} \mathbf{E}$ ) for the two DOA estimators illustrating the potential of better performance with the TR/MIMO radar.

\section{SUMMARY}

A new TR/MIMO radar for target localization is presented, which exploits the spatial diversity arising from multipath and adjusts the probing waveforms to the scattering properties of the medium. As evident from the Monte Carlo simulations, the TR/MIMO radar provides higher accuracy and is more robust to clutter and channel interference than the conventional MIMO radar. The CRBs reinforce the potential gain possible with the TR/MIMO radar.

\section{REFERENCES}

[1] E. Fishler, A. Haimovich, R. Blum, D. Chizhik, L. Cimini, and R. Valenzuela, "MIMO Radar: an idea whose time has come," Proc. of the IEEE Radar Conference, pp. 71-78, Apr. 2004.

[2] J. Li and P. Stoica, MIMO Radar Sig. Processing, J. Wiley, 2008.

[3] Y. Jin, N. O'Donoughue, and J.M.F.Moura, "Time Reversal Adaptive Waveform in MIMO Radar,' Proc. Int'l Conf. on Electromagnetics in Adv. Applications (ICEAA), pp. 741-744, 2010.

[4] Y. Jin, J.M.F. Moura, and N. O’Donoughue, "Time Reversal in Multiple-input Multiple-output Radar," IEEE J. of Selected Topics in Signal Processing, vol. 4, no. 1, pp. 210-225, Feb. 2010.

[5] Harry L. Van Trees, Detection, Estimation, and Modulation Theory: Part III, Wiley-Interscience, New York, USA, 2001.

[6] J. Tabrikian and I. Bekkerman, "Transmission Diversity Smoothing for Multi-target Localization (radar/sonar systems)," Proceedings of ICASSP, vol. 4, pp. iv/1041-iv/1044, Mar. 2005.

[7] F. Foroozan and A. Asif, "Cramér-Rao Bound for Time Reversal Active Array Direction of Arrival Estimators in Multipath Environments," Proceedings of ICASSP, pp. 2646-2649, Mar. 2010.

[8] A. Zeira and A. Nehorai, "Frequency domain CRB for Gaussian processes," IEEE Trans. on Acoustics., Sp. and Sig. Proc., vol. 38, no. 6, pp. 1063 -1066, Jun. 1990. 